\title{
Avaliação da qualidade de vida em soropositivos para o HIV
}

\section{Life quality evaluation in HIV serum-positive individuals}

\author{
Alessandra Ramos CASTANHA² \\ Maria da Penha de Lima COUTINHO² \\ Ana Alayde Werba SALDANHA² \\ Cristiane Galvão RIBEIRO2
}

\begin{abstract}
Resumo
A Síndrome da Imunodeficiência Adquirida tem tratamento e possibilidades efetivas de controle, motivando estudos e intervenções profissionais direcionados para a melhoria da qualidade de vida. Este artigo objetiva avaliar a percepção da Síndrome da Imunodeficiência Adquirida e da qualidade de vida por soropositivos para o Vírus da Imunodeficiência Humana. Participaram deste estudo 91 sujeitos de ambos os sexos. Foi utilizado o teste de associação livre de palavras, cujos dados foram processados pelo software Tri-Deux-Mots e interpretados por meio da análise fatorial de correspondência. A Síndrome da Imunodeficiência Adquirida foi representada como uma doença que pode trazer inúmeras conseqüências psicossociais, profissionais, familiares e orgânicas. A qualidade de vida, por sua vez, foi representada em elementos que possuem características tanto subjetivas quanto objetivas, abrangendo, dessa maneira, diferentes domínios presentes no conceito de qualidade de vida preconizado pela Organização Mundial de Saúde, tais como: domínio psicológico, relações sociais, ambiente e aspectos espirituais. Os anti-retrovirais, apesar de propiciarem uma melhora global, não são suficientes, havendo, ainda, a necessidade de uma abordagem dos transtornos psicológicos para que haja uma qualidade de vida num sentido mais amplo.
\end{abstract}

Unitermos: avaliação; qualidade de vida; representação social; Síndrome da Imunodeficiência Adquirida.

\begin{abstract}
Acquired Immune Deficiency Syndrome has treatment and effective possibilities of control, hence motivating studies and professional interventions towards the improvement of the quality of life. To evaluate the perception of Acquired Immune Deficiency Syndrome and quality of life by Human Immunodeficiency Virus serum-positive individuals. Ninety-one subjects of both genders participated of this study. It was utilized the test of free association of words and the data were processed by the Tri-deux-mots software and interpreted through the factorial analysis of correspondence. The Acquired Immune Deficiency Syndrome was represented as a disease that can cause numerous consequences such as psycho-social, professional, family-and organic-related. The quality of life was represented by elements that have both subjective and objective characteristics, enclosing this way, different matters present in the concept of life quality advocated by the World Health Organization, such as: the psychological aspect, social relations, environment, and spiritual aspects. Even though the anti-retroviral drugs propitiate a general health improvement, they are not enough. Thus there is the necessity of an approach toward the psychological disturbs in order to obtain life quality in a more plentiful way.
\end{abstract}

Uniterms: evaluation; quality of life; social representation; Acquired Immune Deficiency Syndrome.

\section{$\boldsymbol{\nabla \nabla \nabla \nabla}$}

1 Artigo elaborado a partir da dissertação de A.R. CASTANHA, intitulada "Qualidade de vida no contexto da soropositividade para o HIV: um estudo das representações sociais". Universidade Federal da Paraíba, 2005, sob orientação de M.P.L. COUTINHO e co-orientação de C.G. RIBEIRO. Os autores agradecem o apoio financeiro da Coordenação de Aperfeiçoamento de Pessoal de Nível Superior para a realização desta pesquisa.

2 Universidade Federal da Paraíba, Centro de Ciências Humanas Letras e Artes, Departamento de Psicologia. Campus I, Cidade Universitária, 58000-000, João Pessoa, PB, Brasil. Correspondência para/Correspondence to: A.R. CASTANHA. E-mail: <alecas@bol.com.br>. 
Tem havido, no contexto da Síndrome da Imunodeficiência Adquirida (AIDS), uma preocupação crescente com a qualidade de vida das pessoas contaminadas devido ao aumento do tempo de vida proporcionado pela utilização da terapia anti-retroviral e à busca pelos sistemas de saúde, além de métodos de prevenção e controle, de estratégias para facilitar o ajustamento e aumentar o bem-estar dos portadores de Vírus da Imunodeficiência Humana (HIV)/AIDS (Cruz \& Brito, 2005).

Observa-se, no entanto, que a qualidade de vida de soropositivos não está relacionada apenas a uma possibilidade de vida mais longa, pois viver com HIV é se deparar com situações de discriminação, abandono, segregação, estigmatização, falta de recursos sociais e financeiros, ruptura nas relações afetivas e problemas com a sexualidade. Diante desse panorama, enfrentar a doença torna-se cada vez mais problemático e, como conseqüência, a qualidade de vida pode ser comprometida (Galvão, 2002; Galvão, Cerqueira \& Marcondes-Machado, 2004).

É nesse sentido que se insere a importância de estudos na área de qualidade de vida no contexto da AIDS, que contemple uma dimensão psicossociológica. A saúde mental e física, o bem-estar e a qualidade de vida constituem uma preocupação cada vez maior. A saúde depende de fatores individuais e psicológicos, mas, também, de fatores ambientais, socioeconômicos, culturais, históricos e políticos das comunidades e dos países onde os indivíduos estão inseridos (Ramos, 2004).

A Organização Mundial de Saúde (OMS) define qualidade de vida como "a percepção do indivíduo de sua posição na vida, no contexto da cultura e sistema de valores nos quais ele vive e em relação aos seus objetivos, expectativas, padrões e preocupações". Esse é um conceito multidimensional que envolve diferentes domínios (físico, psicológico, social e ambiental), cuja avaliação permite a verificação das dimensões nas quais os tratamentos serão efetivos, podendo direcionar medidas terapêuticas mais adequadas e, provavelmente, diminuir os custos de saúde (TheWHOQOL Group, 1998).

O conceito de qualidade de vida no âmbito da saúde, quando visto no sentido mais amplo, baseia-se na compreensão das necessidades humanas fundamentais, materiais e espirituais e tem no conceito de promoção da saúde seu foco mais relevante (Minayo,

24 Hartz \& Buss, 2000).
O desenvolvimento de estudos na área de qualidade de vida poderá resultar em mudanças nas práticas assistenciais e na consolidação de novos paradigmas do processo saúde-doença. A avaliação de qualidade de vida pode melhorar a relação médico-paciente a partir de uma percepção mais ampla do processo de doença que localize as prioridades e melhore a compreensão, por parte do médico, e a confiança, por parte do paciente.

Na área de pesquisa, os instrumentos de qualidade de vida fornecem informações de como o processo de doença impede o bem-estar do paciente em várias áreas de sua vida, que podem ser direcionadas às políticas de saúde que visam à melhoria da qualidade de vida da população. No campo econômico, a avaliação de qualidade de vida auxilia a definição da relação custo-benefício no impacto que uma doença pode determinar na capacidade produtiva de um sujeito, além de avaliar a qualidade dos recursos de saúde disponíveis (Seidl \& Zannon, 2004).

Dessa forma, o foco desta pesquisa é a qualidade de vida no contexto da AIDS, subsidiada pela teoria das representações sociais (TRS), que permite apreender uma riqueza de elementos figurativos, tais como: cognitivos, ideológicos, normativos, crenças, valores, atitudes, opiniões, imagens, entre outros. Diante da relevância do objeto de estudo para o campo da Psicologia Social e para ações que atendam aos problemas associados à qualidade de vida no contexto da AIDS, este estudo teve por objetivo avaliar a percepção da AIDS e da qualidade de vida por pessoas soropositivas para o HIV, visando intervenções que considerem os aspectos relevantes dos infectados.

\section{Método}

O estudo foi desenvolvido em organizações não-governamentais (ONG) e no Hospital Clementino Fraga, todos especializados no atendimento de soropositivos para o HIV, localizados na cidade de João Pessoa, Paraíba.

Participaram deste estudo 91 sujeitos soropositivos de ambos os sexos, selecionados aleatoriamente, (57\% masculino e 43\% feminino), com média de idade de 38 anos. 


\section{Instrumentos}

Para a coleta dos dados foram utilizados dois instrumentos: um questionário sociodemográfico, visando a uma caracterização da amostra, e a técnica de associação livre de palavras, que, de acordo com Nóbrega e Coutinho (2003), possibilitou acesso aos conteúdos formadores de representações sociais, sem que ocorresse a filtragem da censura à sua evocação. É um instrumento que se apóia sobre um repertório conceitual, permitindo a unificação dos universos semânticos e a saliência de universos de palavras comuns diante de estímulos indutores utilizados no estudo.

Na aplicação da técnica de associação livre de palavras foram utilizados dois estímulos indutores: AIDS e qualidade de vida, previamente definidos, tendo como pressuposto o objeto social investigado. Foi estipulado o tempo máximo de um minuto para evocação das palavras associadas a cada estímulo indutor.

\section{Procedimentos}

Após aprovação pelo Comitê de Ética em Pesquisa, do Centro de Ciências da Saúde da Universidade Federal da Paraíba, realizou-se um estudo-piloto com o intuito de verificar a adequação do instrumento, confirmando sua validade semântica; em seguida, efetuou-se o estudo definitivo. Foi feita uma apresentação dos pesquisadores aos grupos, na qual se explicitaram os objetivos do estudo, garantindo o anonimato e a confiabilidade das respostas, indicando-Ihes que seriam analisadas no seu conjunto; posteriormente, iniciou-se a aplicação dos instrumentos de forma coletiva.

\section{Resultados}

Os dados coletados pela técnica de associação livre foram processados pelo software Tri-Deux-Mots (Cibois, 1991), versão 2.2, que permite a visualização gráfica tanto das variáveis fixas (sexo e idade) como das de opinião (conhecimento prático enunciado pelos sujeitos diante do estímulo indutor), analisadas pela análise fatorial de correspondência (AFC).
O somatório de todas as palavras evocadas $(\Sigma=1.047)$ pelo coletivo dos participantes $(n=91)$ que compõem a amostra da pesquisa, relativas a cada estímulo indutor, é analisado em função da freqüência e da importância relativa às variáveis fixas (sexo e idade), determinando o espaço fatorial ou gráfico

A análise fatorial de correspondência é um procedimento estatístico que consiste em distinguir os vínculos estabelecidos entre as características dos participantes que constituem um grupo e suas respostas a uma determinada questão. Desse modo, verificaram-se grupos diferenciados quanto ao conteúdo das representações e de sua estrutura, explicitadas pelas pessoas soropositivas que se colocam em posições, simetricamente, opostas em função das variáveis sexo e idade (Figura 1).

O fator 1 (F1, negrito) explica 40,7\% da variância total das modalidades (palavras-resposta) processadas; o fator 2 (F2, itálico) explica 31,7\%, perfazendo um total de $72,4 \%$ da variância total, possuindo, portanto, parâmetros estatísticos com consistência interna e fidedignidade, tendo em vista pesquisas realizadas no âmbito das RS (Castanha, 2005; Castanha, Coutinho \& Saldanha, 2005; Nóbrega \& Coutinho, 2003). O espaço fatorial, constituído dos dois primeiros fatores (F1 e F2)

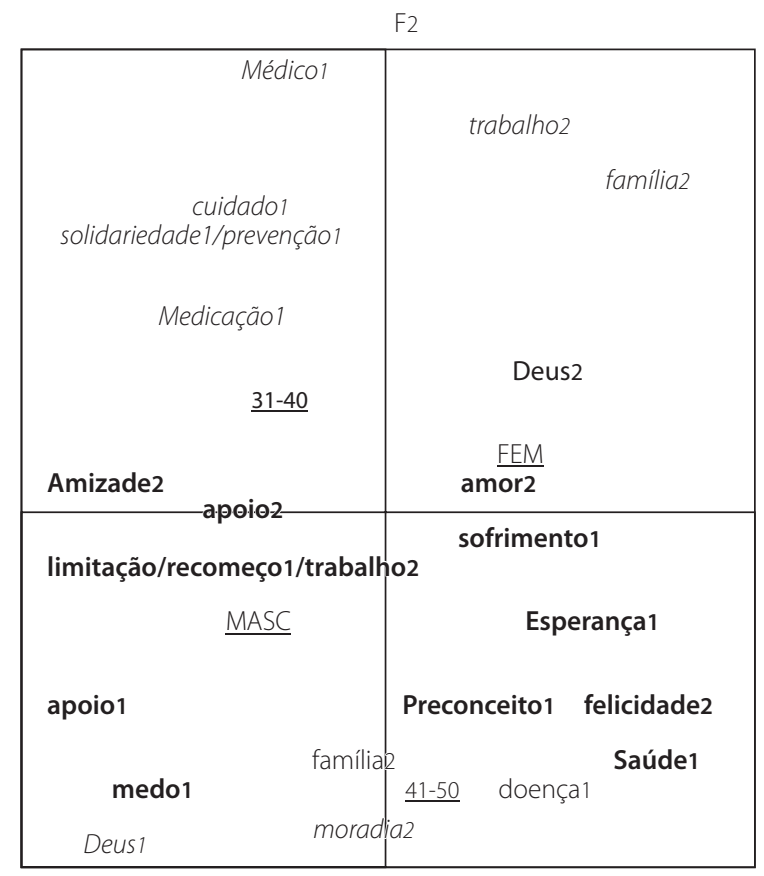

Figura 1. Plano fatorial dos eixos 1 e 2 das representações sociais da AIDS e da qualidade de vida. 
e delimitado pelas respostas aos dois estímulos indutores (1 = AIDS e 2 = qualidade de vida), revela a existência de agrupamentos representacionais ou, o que se denomina em estatística, de nuvens, que evidenciam as semelhanças e as diferenças nos conteúdos e na estrutura das representações dos soropositivos (Figura 1).

Observa-se que a Figura 1 destaca com clareza dois campos semânticos configurados em oposição ao fator 1. No eixo à esquerda, em negrito, encontram-se aglomeradas as representações que caracterizam o grupo dos homens. Para esse grupo, a AIDS (estímulo 1) é representada como limitação, medo, recomeço e apoio. A qualidade de vida (estímulo 2), por sua vez, é representada como trabalho, apoio e amizade. Inversamente, nesse mesmo fator, à direita, configura-se o campo semântico elaborado pelas mulheres que associam a AIDS (estímulo 1) aos elementos de sofrimento, preconceito, esperança e saúde. Já a qualidade de vida (estímulo 2) foi associada aos elementos de felicidade e Deus.

Com relação ao fator $F 2$, linha vertical, os grupos compreendidos na faixa etária de 31 a 40 anos e 41 a 50 anos, de ambos os sexos, encontram-se, diametralmente, distanciados, o que indica discordâncias representacionais. Na margem superior localizam-se as representações dos sujeitos soropositivos de ambos os sexos compreendidos na faixa etária de 31 a 40 anos, associadas à: prevenção, cuidado, médico, medicação e solidariedade, no que diz respeito à AIDS (estímulo 1); e trabalho e família no que diz respeito à qualidade de vida (estímulo 2). Na margem inferior, destacam-se as modalidades representacionais dos sujeitos soropositivos de ambos os sexos compreendidos na faixa etária de 41 a 50 anos, configuradas nos elementos: doença e Deus (AIDS); e moradia e família (qualidade de vida).

Os campos semânticos inseridos no fator 1 apontam a existência de representações similares entre esses dois grupos, dentre as quais podemos citar: doença, médico, medicação (AIDS) e família (qualidade de vida). Esses elementos são figurativos por representarem o que há de consensual nesses dois grupos, sendo chamados também de elementos do núcleo central. Além desses elementos consensuais, observam26 se alguns elementos distintos. No grupo de 31 a 40 anos, de ambos os sexos, surgiram os elementos cuidado, solidariedade, e prevenção (AIDS); e trabalho (qualidade de vida). Já no grupo de 41 a 50 anos emergiram os elementos Deus (AIDS); e moradia (qualidade de vida).

Denota-se, por meio desses resultados, que as representações sociais dos soropositivos, tanto homens como mulheres, estão dimensionadas em dois eixos: objetivo e subjetivo, respectivamente. Essa característica pode ser explicada pela diferença de gênero, que influencia a percepção e o comportamento diante da vida.

No grupo de homens (primeiro grupo) há um predomínio de elementos objetivos, em que os atores sociais representaram a AIDS (estímulo 1) como uma doença que causa medo, que acarreta algumas limitações, exigindo um novo recomeço de vida, sendo importante que haja apoio. No grupo de mulheres (segundo grupo) há um predomínio de elementos subjetivos, representando a AIDS como uma doença na qual se vivencia o preconceito, que acarreta muito sofrimento e para a qual se busca uma esperança de saúde.

Apesar da evolução terapêutica e de seu reflexo direto na reinterpretação da AIDS, observa-se, ainda hoje, que ela continua sendo associada à morte e a todo o sofrimento que lhe é pertinente. Segundo Tura (1998), a morte está nitidamente apresentada como elemento do núcleo central das representações sociais da AIDS e é organizadora de várias cognições. A morte é vista como terrível, premeditada, lenta, mobilizadora do medo, e discriminadora do outro que não se previne. Mesmo com o advento da terapêutica anti-retroviral, a AIDS ainda é uma doença potencialmente letal e conviver com a idéia de morte é sempre um fator gerador de estresse e sintomas psíquicos.

O sofrimento causado pela AIDS pode ser proveniente de diversos fatores, tais como: o diagnóstico, o processo do adoecer, a iminência de morte, a discriminação, as limitações e as perdas (Malbergier, 2000).

Com relação às limitações provocadas pelo HIV/AIDS, em vista do sofrimento provocado pela influência do medicamento e da própria evolução da doença, elas estão, muitas vezes, relacionadas ao aspecto psicossocial em que esses indivíduos se encontram. 
Apesar de a terapêutica atual ter prolongado a vida dos pacientes infectados e melhorado bastante sua qualidade de vida, viver com AIDS impõe várias limitações: profissionais, de relações afetivas, dificuldade de manter relacionamentos sexuais, decisão de ter ou não filhos (Malbergier, 2000). A incapacidade trazida pelo adoecimento significa não corresponder às expectativas da família e da sociedade, concorrendo para frustração e interrupção de um projeto de vida pessoal, familiar e profissional.

Ainda nesse sentido, pode-se descrever uma série de alterações relacionadas às perdas físicas, psicológicas e sociais que o portador do HIV sofre, modificando os aspectos relacionados à qualidade de vida. A auto-estima diminui e o indivíduo fica sem crenças ou planos futuros, tomado pela tristeza e depressão. Nesse sentido, a intervenção psicológica é fundamental na tentativa de minimizar o sofrimento psíquico, dando suporte à adaptação às mudanças decorrentes da evolução da doença (Arpinelli, Visona, Bruno, De Carli, \& Apolone, 2000).

A AIDS acarreta uma diversidade de sintomas dentre os quais pode-se citar os transtornos psicológicos e a deteriorização da qualidade de vida. Os anti-retrovirais, apesar de propiciarem uma melhora global dos pacientes, não são suficientes, existe as limitações, havendo, pois, a necessidade de uma abordagem desses transtornos para que haja uma qualidade de vida num sentido mais amplo (Martín et al., 2002).

No que diz respeito ao preconceito, Sousa (2001) afirmou que além da AIDS ser uma doença com graves conseqüências físicas e psicológicas, ela constitui-se também como um fenômeno de natureza social, acompanhado de processos de segregação social baseados em estigmas socialmente construídos e intimamente ligados às representações sociais da doença. Apesar da comprovação científica sobre os meios de transmissão da doença, ainda existe muito medo, preconceito e discriminação em relação aos portadores do HIV/AIDS, perpetuando aspectos levantados por Saldanha, Figueiredo e Coutinho (2004), tais como: o medo irracional, aspectos psicossociais desfavoráveis evidenciados por desinformações, discriminações e atitudes negativas.

Dessa maneira, contrair o vírus HIV implica uma transgressão que favorece uma estereotipia de acusações e culpa de caráter subjetivo e revestida de caráter da alteridade, cujos comportamentos são representados como ameaças à sociedade por serem portadores de uma doença tão temida. Esses comportamentos seriam a conseqüência de um comportamento sancionado negativamente por certos grupos da sociedade, como forma de punição moral pelo pecado (Camargo, 2003).

As mulheres representaram a AIDS como uma doença que carrega com ela o preconceito, sendo a esperança e o suporte ancorado na religiosidade, objetivado na figura de Deus, uma das maneiras de enfrentá-la. A AIDS é vista também como um recomeço: após o impacto sofrido pelo diagnóstico, vem a necessidade da reestruturação e manutenção da vida em seus vários papéis.

A construção de uma nova identidade após o adoecimento e o retorno à normalidade - que não é saúde - constitui-se um processo dinâmico e complexo, que consiste em assimilar e produzir, de forma ativa, conhecimentos e formas de ação. Com o passar do tempo a doença é integrada às esferas da vida como um aspecto a mais, sem que isso implique sua banalização. "Trata-se de um tipo de gestão racional da doença, em que as angústias despertadas pelo diagnóstico são canalizadas para as questões do cotidiano" (Saldanha, 2003, p.45).

Percebeu-se, ainda em relação aos participantes, independentemente do sexo, que a qualidade de vida (estímulo 2) foi representada de forma objetiva e subjetiva, respectivamente. No grupo dos homens, a qualidade de vida está associada ao trabalho e ao apoio advindo da amizade. Já no grupo das mulheres, observa-se um predomínio de elementos subjetivos ao representarem a qualidade de vida associada ao amor, à felicidade e à figura de Deus, advinda na crença da religiosidade.

A qualidade de vida foi percebida como sendo composta de fatores objetivos e subjetivos. Essas características foram encontradas também em estudos sobre a subjetividade do bem-estar, que têm demonstrado que não são apenas as condições sociodemográficas (estado civil, idade, sexo, renda, etnia, etc.) que definem a sensação de bem-estar das pessoas, mas uma combinação de processos subjetivos próprios de cada indivíduo, isto é, as estruturas internas da pessoa 
determinam a forma como são percebidos e internalizados os eventos externos, o que refletirá na auto-avaliação do seu estado de bem-estar (Diener, Suh, Lucas \& Smith, 1999; Gouveia, Chaves, Dias, Gouveia \& Andrade, 2003).

No contexto da soropositividade, o elemento trabalho se propaga em dois eixos: um de caráter mais positivo, que favorece uma melhor qualidade de vida ao propiciar melhores condições financeiras e oferecer estabilidade e credibilidade, e ocupação psicossocial, atribuindo ao indivíduo perante a sociedade o status de produtivo; e um outro eixo de caráter mais negativo, no qual o trabalho é associado à diminuição e/ou perda da capacidade. Essas premissas corroboram as investigações desenvolvidas por Laurell (1989), nas quais o trabalho encontra-se vinculado a vários atributos e funções morais. É por meio dele que o indivíduo atribui significado e sentido à própria existência. Tendo o trabalho um sentido tão amplo e estruturante na vida das pessoas, qualquer limitação que impeça de exercer uma atividade produtiva provoca alterações profundas na vida, tornando o indivíduo doente mais sujeitado e sem direitos, afetando diretamente a qualidade de vida daquele que, geralmente, é aposentado ou está desempregado em função de sua condição de soropositivo.

A qualidade de vida no contexto da soropositividade também perpassa pelo apoio e suporte social, que pode advir da família, dos amigos e da própria religião. O fortalecimento da rede social de apoio é uma das novas questões de natureza psicossocial vivenciadas por pessoas soropositivas (Seidl, Zannon \& Truccoli, 2005).

No entanto, apesar dos avanços de pesquisas no contexto da soropositividade, as dificuldades oriundas do contexto sociocultural e familiar ainda persistem, haja vista o preconceito existente em torno da doença que faz com que os portadores se sintam culpados diante do que não podem reverter - culpa que a família, os amigos e a sociedade reforçam com cobranças, discriminação, isolamento e omissão. 0 suporte social é importante na diminuição do risco de doença e de mortalidade associados a certas doenças, e melhora o prognóstico e a qualidade de vida (Teixeira,

28 2000).
Os dados de modo geral mostram uma relação direta entre as representações sociais da AIDS e da qualidade de vida, deixando evidente como a soropositividade influencia diretamente na qualidade de vida. As mulheres, ao mesmo tempo em que representaram a AIDS em sofrimento, preconceito e esperança, representaram a qualidade de vida em felicidade, amor e Deus. Já os homens, ao mesmo tempo em que representaram a AIDS em medo, apoio, recomeço e limitação, representaram a qualidade de vida em apoio, amigos e trabalho.

Os grupos de sujeitos soropositivos compreendidos na faixa etária entre 31 e 40 anos e 41 e 50 anos não estão divididos em eixos, possivelmente por estarem constituídos por sujeitos de ambos os sexos, percebendo-se assim tanto elementos objetivos como subjetivos em ambos os grupos, independentemente da faixa etária.

O primeiro grupo (31 a 40 anos) representou a AIDS como uma doença que deve ser prevenida, cujo cuidado é feito pelo acompanhamento médico e medicamentoso, havendo ainda a necessidade de solidariedade dos integrantes de sua rede social. O segundo grupo (41 a 50 anos) representou a AIDS como uma doença que pode ser enfrentada pela esperança e pelo apoio que pode vir da religiosidade, objetivada na figura de Deus.

Dados semelhantes foram encontrados em estudo realizado por Camargo (2000) com estudantes universitários sobre a sexualidade e as representações sociais da AIDS, no qual foram identificados como elementos supostamente centrais no campo das representações sociais da AIDS: sexo, prevenção, drogas, doença e morte; e como elementos possivelmente periféricos: discriminação, promiscuidade, descuido, tristeza, preconceito e solidariedade. $\mathrm{O}$ autor afirmou, ainda, que duas noções passam a conviver quando se pensa em AIDS: doença mortal e doença sexualmente transmissível que pode ser evitada com a prevenção (sobretudo com a utilização de preservativos).

Os atores sociais da pequisa mencionada acima representaram a AIDS como uma doença com toda uma gama de cuidados médicos e medicamentosos necessários para a manutenção da vida, indicando a luta dos soropositivos para melhorar a qualidade de vida. 
Com relação aos elementos esperança, apoio e Deus, muitos estudos abordaram as diferentes estratégias pelas quais as religiões reinterpretaram a experiência da doença e modificaram a maneira pela qual o doente e o meio social definem o problema. Segundo Gir (1997), as religiões são criadas como uma estrutura para um sistema de crenças, valores, códigos de condutas e rituais e apresentam os componentes de fé, ou crença, esperança e amor. O indivíduo com crenças religiosas sente em si mais força, seja para suportar as dificuldades da existência seja para vencê-las. Dessa maneira, os soropositivos encontram na crença religiosa um grande auxílio para suportar as privações e as angústias impostas pela doença.

Observou-se nesses dois grupos que os mais jovens (31 a 40 anos) estão mais ligados às questões concretas da AIDS e da busca, não de uma cura milagrosa, mas da manutenção da vida através de tratamento médico/medicamentoso, contando com o apoio não do divino, mas da solidariedade das pessoas que os cercam em suas vidas diárias. Já os mais velhos (41 a 50 anos) estão mais voltados à religiosidade, com a esperança e a fé de cura para essa doença que tanto os aflige. A característica encontrada no último grupo pode ser considerada positiva, quando servir de apoio, ou negativa, por poder levar a uma baixa adesão ao tratamento e, conseqüentemente, a uma baixa qualidade de vida.

A religião pode surgir como apoio, representando uma importante rede de suporte emocional, mas pode também atuar de forma negativa na vida de soropositivos. Saldanha (2003) observou em mulheres soropositivas um aumento, após a contaminação, da procura pela religião, através da qual alegaram receber grande conforto e esperança de cura. A autora afirma ainda que desde que as crenças não se tornem exacerbadas, prejudicando ou interrompendo o tratamento, a fé e a esperança de cura adquiridas contribuem para uma melhora no quadro geral do paciente.

No que diz respeito à qualidade de vida para o grupo dos sujeitos soropositivos compreendidos na faixa etária de 31 a 40 anos, de ambos os sexos, ela perpassa pelo trabalho e pela família. Já para o grupo dos soropositivos compreendidos na faixa etária de 41 a 50 anos, a qualidade de vida perpassa pela moradia e também pela família.
Observou-se, nesses grupos, que a qualidade de vida está relacionada a questões objetivas, tais como: o trabalho e a moradia, e ressaltou-se que a grande maioria dos atores sociais possui condição financeira precária que influencia diretamente na relevância dos fatores na qualidade de vida dos infectados pelo HIV. Observou-se, também, a importância da família como sendo um preditor de uma melhor qualidade de vida.

Nesse sentido, Brook (1999) afirmou que o suporte social familiar está positivamente associado ao bem-estar psicológico, e que o suporte dos amigos é útil para a mudança de hábitos de vida, nomeadamente no abandono de comportamentos sexuais de risco e do consumo de drogas, o que, conseqüentemente, contribuiu não só na prevenção, mas também na adesão ao tratamento. O modo como as famílias se adaptam à doença é um reflexo do seu relacionamento íntimo, de suas histórias e do contexto sociocultural em que estão inseridas. Quando a estrutura familiar apresentou um relacionamento satisfatório, o nível de receptividade e de compreensão se deu mais facilmente, trazendo ao membro que se encontrava fragilizado diante da doença um fortalecimento por meio das relações de ajuda e afeto (Saldanha, 2003).

O choque da família confirmou a fragilidade dos vínculos, levando à rejeição o paciente e negando-lhe qualquer tipo de ajuda ou auxílio, tratando-o como culpado de seu estado, considerando a doença como um castigo devido ao comportamento ou opção errada de vida. Outras famílias, ao contrário, superprotegem, tentando evitar o sofrimento, reforçando um comportamento onipotente, e dificultando o amadurecimento emocional do doente (Joffe, 2002).

Uma das maiores dificuldades ou limitações da nossa pesquisa, e que se observou também na literatura consultada, foi a evidência de que a discriminação sofrida pelos soropositivos ocorre, muitas vezes, no próprio núcleo familiar, o que dificulta a configuração de uma rede de apoio que propicie melhor qualidade de vida.

\section{Conclusão}

Por meio das representações sociais apreendidas neste estudo pode-se ter acesso às crenças, às interpretações, ao conjunto de relações sociais. Essas 
representações ultrapassaram o aspecto biológico e atingiram os aspectos psicossociais nos quais o preconceito, a segregação, o estigma e a inserção social ainda estão bastante presentes no contexto da soropositividade.

A AIDS foi representada como uma doença que pode ocasionar inúmeras conseqüências psicossociais, profissionais, familiares e orgânicas, atingindo assim o ser humano em sua globalidade, e repercutindo em vários aspectos da vida, como na produtividade, na capacitação social e na qualidade de vida.

A qualidade de vida, por sua vez, foi representada em elementos que possuem características tanto subjetivas quanto objetivas: trabalho, moradia, apoio, amizade, felicidade, Deus e família. Essas representações estão de acordo com o conceito preconizado pela Organização Mundial de Saúde, em que a qualidade de vida se refere à percepção do indivíduo de sua posição na vida, no contexto da cultura e no sistema de valores nos quais ele vive e em relação aos seus objetivos, expectativas, padrões e preocupações (The WHOQOL Group, 1998), abrangendo, assim, diferentes domínios, tais como o psicológico, o das relações sociais, ambiente e aspectos espirituais.

A presente pesquisa não esgota a possibilidade de futuros estudos sobre o tema. No entanto espera-se que ela possa contribuir na formulação de novos questionamentos acerca da qualidade de vida de soropositivos para o HIV, de forma a preencher algumas lacunas até agora existentes, possibilitando melhor compreensão desse fenômeno biopsicossocial.

\section{Referências}

Arpinelli, F., Visona, G., Bruno, R., De Carli, G., \& Apolone, G. (2000). Health-related quality of life in asymptomatic patients with HIV. Evaluation of the SF-36 health survey in Italian patients. Pharmacoeconomics, 18 (1), 63-72.

Brook D. W. (1999). Coping strategies of HIV positive and HIV negative female injection drug users: a longitudinal study. AIDS Education and Prevention, 11 (5), 373-88

Camargo B. V. (2000). Sexualidade e representações sociais da AIDS. Revista de Ciências Humanas, 3 (Número temático: Representações sociais e interdisciplinariedade), 97-110.

Camargo, B. V. (2003). A televisão como vetor de difusão de informações sobre a AIDS. In Representações sociais: abordagem interdisciplinar (pp.130-152). João Pessoa: 30 UFPB.
Castanha, A. R. (2005). A qualidade de vida no contexto da soropositividade para o HIV: um estudo das representações sociais. Dissertação de mestrado não-publicada, Centro de Ciências Humanas, Letras e Artes, Universidade Federal da Paraíba, João Pessoa.

Castanha, A. R., Coutinho, M. P. L., \& Saldanha, A. A. W. (2005). Depressão e soropositividade para o HIV: dois fenômenos em extensão. In M. P. L. Coutinho \& A. A. W. Saldanha (Orgs.), Representação social e práticas de pesquisa (pp.173-190). João Pessoa: UFPB.

Cibois, P. (1991). L'analyse factorielle: collecion "Que sais-je?" (3a. ed.). Paris: Puf.

Cruz, E. F., \& Brito, N. (2005). Fios da vida: tecendo o feminino em tempos de AIDS. Brasília: Ministério da Saúde.

Diener, E., Suh, E. M., Lucas, R. E., \& Smith, H. L. (1999). Subjective well-being. Three decades of progress. Psychological Bulletin, 125 (2), 276-302.

Galvão, M. T. G. (2002). Aplicação do instrumento HAT - QOL para análise da qualidade de vida de mulheres c/ infecção pelo HIV, ou com AIDS esua correlação com as variáveis sócio - demográficas, epidemiológicas e clinicas. Dissertação não-publicada, Centro de Pós-Graduação em Doenças Tropicais, Faculdade de Medicina de Botucatu, Universidade Estadual Paulista, Botucatu.

Galvão, M. T. G., Cerqueira, A. T. A. R., \& Marcondes-Machado, J. (2004). Avaliação da qualidade de vida de mulheres com HIV/AIDS através do HAT-QOL. Cadernos de Saúde Pública, 20 (2), 430-437.

Gir, E. (1997). A sexualidade e a mulher portadora do vírus da imunodeficiência humana tipo 1 (HIV-1). Tese de doutorado não-publicada, Escola de Enfermagem de Ribeirão Preto, Universidade de São Paulo, Ribeirão Preto.

Gouveia, V. V., Chaves, S. S. S., Dias, M. R., Gouveia, R. S. V., \& Andrade, P. R. (2003). Valores humanos y salud general: Aportaciones desde la psicología social. In M. A. Vidal (Org.), Psicosociología del cuidado (pp.19-38). Moncada: CEU.

Joffe, H. (2002). "Eu não, o meu grupo não": representações sociais transculturais da AIDS. In P. Guareschi \& S. Jovchelovitch (Orgs.), Textos em representações sociais (pp.297-322). Petrópolis: Vozes.

Laurell, C., \& Noriega, M. (1989). Processo de produção e saúde. Trabalho e desgaste operário. São Paulo: HUCITEC.

Malbergier, A. (2000). AIDS e Psiquiatria: um guia para os profissionais de saúde. Rio de Janeiro: Revinter.

Martín, S. I., Cano, M. R., Pérez de Ayala, P., Aguayo, C. M., Cuesta, F., Rodríguez, P., \& Pujol de la Llave, E. (2002). Calidad de vida, aspectos psicológicos y sociales en pacientes con infección VIH avanzada. Anales de Medicina Interna, 19 (8), 396-404.

Minayo, M. C. S., Hartz, Z. M. A., \& Buss, P. M. (2000). Qualidade de vida e saúde: um debate necessário. Ciência \& Saúde Coletiva, 5 (1), 7-18.

Nóbrega, S. M., \& Coutinho, M. P. L. (2003). O teste de associação livre de palavras. In M. P. L. Coutinho (Org.), 
Representações sociais: abordagem interdisciplinar (pp.67-77). João Pessoa: UFPB.

Ramos, N. (2004). Psicologia clínica e da saúde. Lisboa: Universidade Aberta.

Saldanha, A. A. W. (2003). Vulnerabilidade e construções de enfrentamento da soropositividade ao HIV por mulheres infectadas em relacionamento estável. Tese de doutorado não-publicada, Pós-Graduação em Psicologia, Universidade de São Paulo, Ribeirão Preto.

Saldanha, A. A. W., Figueiredo M. A. C., \& Coutinho, M. P. L. (2004). Atendimento psicossocial à AIDS: a busca pelas questões subjetivas. DST-Jornal Brasileiro Doenças Sexualmente Transmissíveis, 16 (3), 84-91.

Seidl, E. M. F., \& Zannon, C. M. L. C. (2004). Qualidade de vida e saúde: aspectos conceituais e Metodológicos. Cadernos Saúde Pública, 20 (2), 580-588.

Seidl, E. M. F., Zannon, C. M. L.C., \& Truccoli, B. T. (2005). Pessoas vivendo com HIV/AIDS: enfrentamento, suporte social e qualidade de vida. Psicologia: Reflexão e Crítica, 18 (2), 188-195.
Sousa, M. M. O. (2001). SIDA: e a vida continua. Lisboa: Associação Portuguesa de Enfermeiros.

Teixeira, P. R. (2000). Experiências de adesão ao tratamento anti-retroviral em São Paulo. In P. R. Teixeira, V. Paiva \& E. Shimma (Orgs.), Tádifícil deengolir? Experiências de adesão ao tratamento anti-retroviral em São Paulo (pp.115-123). São Paulo: NepAIDS.

The WHOQOL Group. (1998). The World Health Organization quality of life assessment (WHOQOL): development and general psychometric properties. Social Science and Medicine, 46 (15), 69-85.

Tura, L. F. R. (1998). AIDS e estudantes: a estrutura das representações sociais. In D. Jodelet (Org.), AIDS e representações sociais: a busca de sentidos (pp.121-154). Natal: EDUFRN.

Recebido em: 19/7/2005

Versão final reapresentada em: 1/8/2006

Aprovado em: 15/9/2006 
